\title{
Editorial
}

\section{Variability of Atmospheric, Oceanic, and Hydrological Phenomena}

\author{
Leonard J. Pietrafesa, ${ }^{1}$ Alan Blumberg, ${ }^{2}$ and Xiaofeng $\mathrm{Li}^{3}$ \\ ${ }^{1}$ The Coastal Carolina University, Conway, AR, USA \\ ${ }^{2}$ The Stevens Institute of Technology, Hoboken, NJ, USA \\ ${ }^{3}$ The National Oceanic \& Atmospheric Administration, College Park, MD, USA
}

Correspondence should be addressed to Leonard J. Pietrafesa; ljpietra@ncsu.edu

Received 27 December 2015; Accepted 27 December 2015

Copyright ( $\odot 2016$ Leonard J. Pietrafesa et al. This is an open access article distributed under the Creative Commons Attribution License, which permits unrestricted use, distribution, and reproduction in any medium, provided the original work is properly cited.

The temporal and spatial scales of atmospheric, oceanic, and hydrologic environmental phenomena span the time frame from isolated events to that of subseasonal variability in the Earth's interactively coupled atmospheric, oceanic, and hydrological systems globally. There are significant associated implications for global human and ecological systems and these have become an emerging topic around which issues of societal and economic value and sustainability can be examined and used for societal response and planning. In this special issue, massive observational data sets and mathematical modeling tools have been applied to couple the observed and/or modeled environmental processes to societal impacts. Examples include coastal and inshore flooding events, normally associated with tropical cyclones which, because of sea level rise, could extend into the winter season when extratropical cyclones are present and also include a higher frequency of flood events in the Americas, Europe, Asia, Africa, and Australia. The societal implications of these events are huge and other evolving and emerging phenomena in kind have been revealed.

The topics covered in this special issue generally include the following: feasibility and "proof-of-concept" studies utilizing diagnostic and prognostic tools; pilot to mature studies relevant to the use of atmospheric, oceanic, and hydrological data sets including in situ and remotely sensed data sets, to drive societally relevant research; modern analytics and informatics including utilizing environmental and socioeconomics data; numerical modeling results focusing on leveraging interoperability and knowledge management; value assessments of environmental data, including investments in satellite and other technologies; studies using new methodological paradigms in environmental research; and environmental information that provides enabling capacity for environmental managers and society. The specific topics include the following: rainfall and cloud dynamics; flood hazard mapping by using geographic information systems and hydraulic models; the detection of abrupt changes in runoff and river discharge; the historical and future spatial and temporal variability of precipitation; the suitability and sustainability of water harvesting; the dynamics of a typhoon and a related high ozone episode; the forecasting of oceanic surface currents via the assimilation of radar data in a numerical model; the impact of wind stress on the global heat transport, thermohaline circulation, and oceanic instability; the onset and evolution of hydrological drought in human disturbed areas; projections of future drought hazard areas based on climate change scenarios; and the effects of climate and anthropogenic activity on hydrologic riverine features.

This special issue was intended to present scientific breakthrough results which are expected to reveal the couplings of environmental event scale with subseasonal variability scale to the implications for society at local to regional and global scale environments. It has succeeded in doing so.

\section{Leonard J. Pietrafesa Alan Blumberg Xiaofeng $L i$}



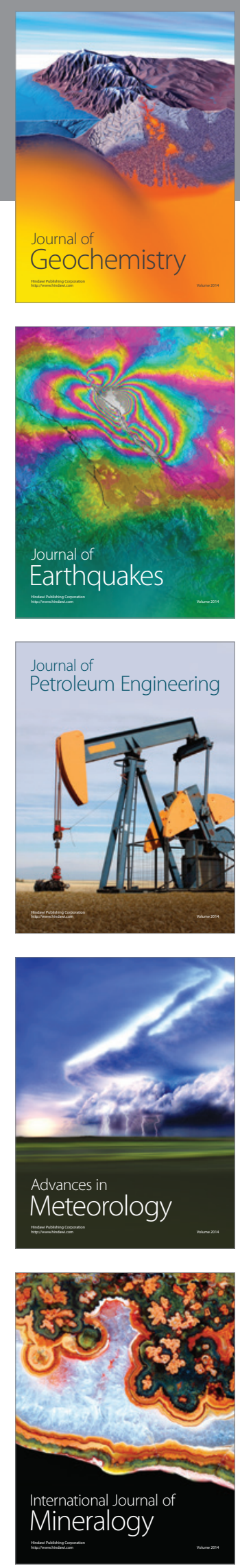
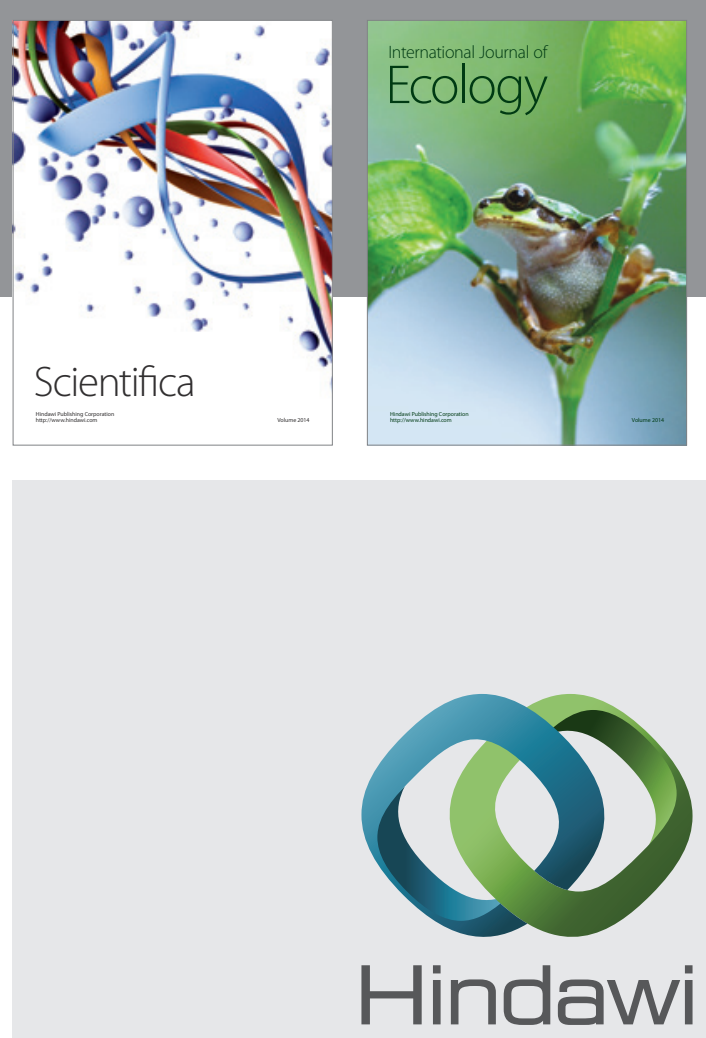

Submit your manuscripts at

http://www.hindawi.com
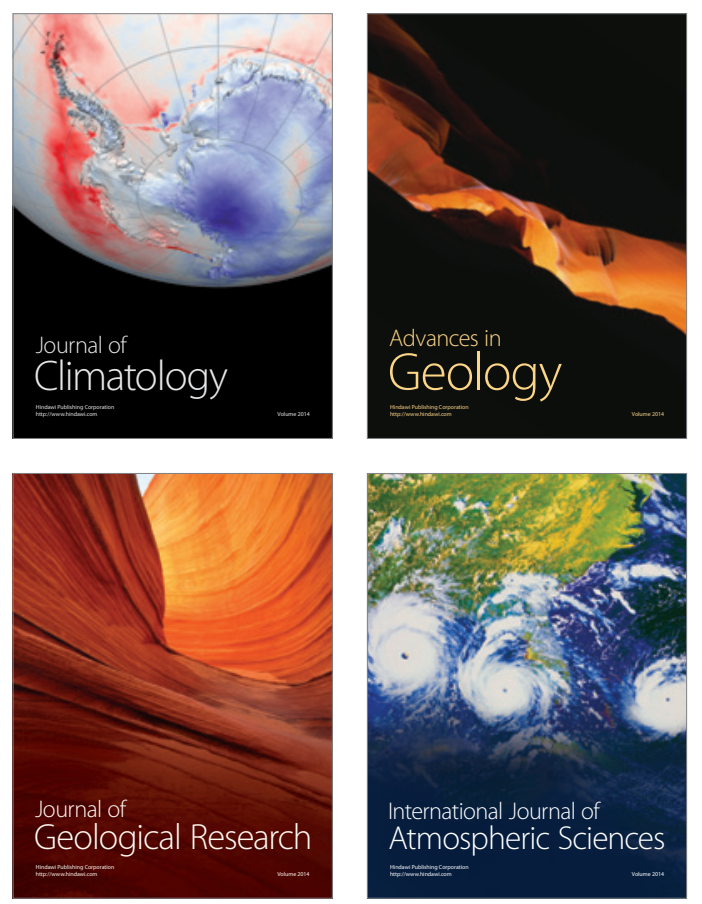

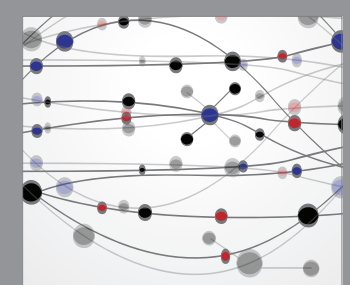

The Scientific

\section{World Journal}
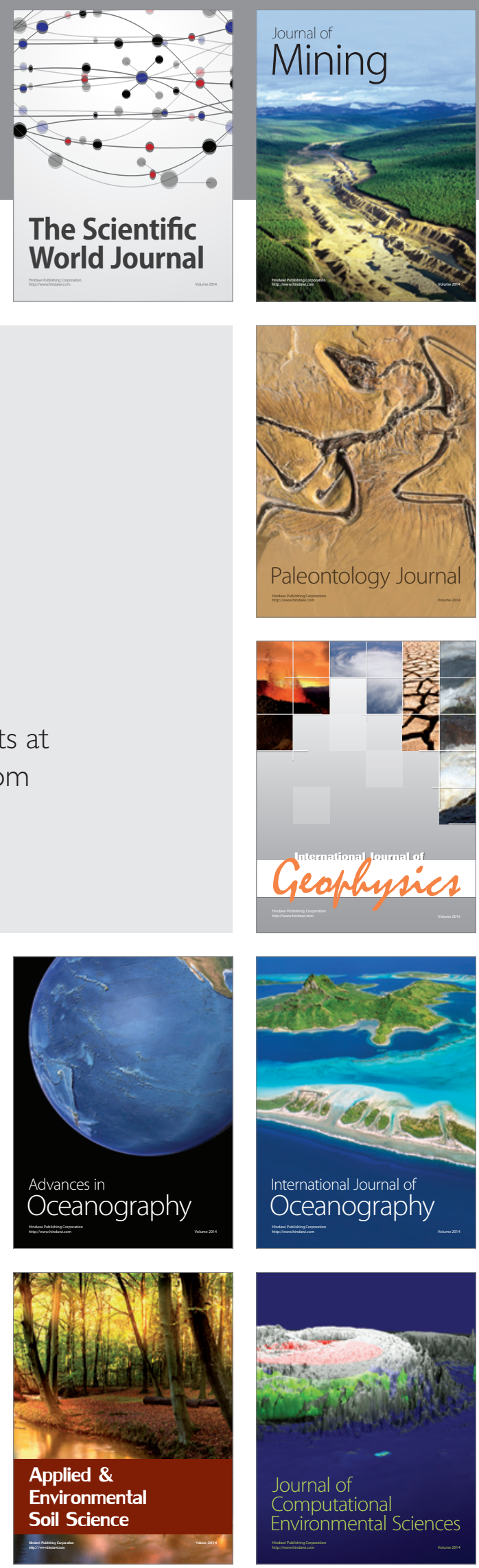